0038-1098(95)00636-2

\title{
STRONG COUPLING CHARACTERISATION OF QUASI-1D POLARONS IN CYLINDRICAL QW-WIRES
}

\author{
A. Erçelebi and R.T. Senger \\ Department of Physics, Bilkent University, 06533 Ankara, Turkey
}

(Received 20 June 1995 by P. Burlet)

\begin{abstract}
We retrieve, within the strong-coupling theory, the quasi-one dimensional analog of the standard optical polaron relevant to a cylindrical quantum well wire. Under the assumption of perfect confinement the ground state binding energy, effective polaronic mass and the phononcoupling-induced potential well profiles are given as a function of the wire radius and the electron-phonon interaction strength.
\end{abstract}

Keywords: A. quantum well, D. electron-phonon interaction.

\section{INTRODUCTION}

THE STUDY of quantum well-heterostructure-type systems continues to be an attractive field from both theoretical and experimental viewpoints. Such systems with reduced dimensionality have become important as a basis for novel devices, owing to the possibility of tailoring their electronic and optical properties. The impressive progress achieved in microfabrication technology (such as molecular beam epitaxy, lithographic and etching techniques) has created a variety of opportunities for the fabrication of new semiconductor structures. Of particular interest is the quantum well wire (QWW) configuration based on the confinement of electrons in a thin semiconducting wire where the motion is quantised in the transverse directions normal to its length. Since their early prediction [1] and subsequent fabrication [2-5], there has appeared quite a large interest in phonon-coupling-induced effects and polaronic properties of one-dimensionally confined electrons. Some considerable amount of the literature published within this context has been devoted to the interaction of electrons with bulk-like LO-phonons and the study of the relevant polaron properties [6-13]. The common prediction led by these works is that in quantum wires where the electrons are fundamentally quasi-one dimensional (Q1D) the polaronic binding is far deeper than in comparable quasi-two dimensional systems. In other words, high degrees of confinement (as realised in thin wires) led to a pseudo-enhancement in the effective electronphonon coupling, which in turn brings about the possibility that, in spite of weak polar coupling as in GaAs for instance, the polaron problem may also have a strong-coupling counterpart coming from confinement effects. This salient feature can be more prominent in II-VI compound semiconductors or in alkali halides where the relevant coupling strengths are almost an order of magnitude larger or even much stronger than those in III-V materials. We thus feel that for not too weak and pseudo-enhanced electronphonon coupling, the strong-coupling polaron theory should not be accounted for as a totally academic formalism but may provide some insight into the study of polarons in confined media consisting of materials of somewhat stronger polar crystals.

In this context we therefore find it worthwhile to retrieve the polaron problem in a cylindrical wire within the framework of the strong-coupling theory. In fact, a similar problem has been treated previously for a parabolic boundary potential providing a Q1Dtubular confinement where the free optical polaron properties have been derived as a function of the effective reduction in the dimensionality $[10,12]$. For completeness we review the same problem where now we refer to the case of an electron perfectly confined within a cylindrical boundary with infinite potential.

For the present we refrain from including the coupling of the electron to the confined phonon modes as well as interface SO-phonons and adopt the so-called bulk-phonon approximation, where a laterally 
confined electron is visualised as interacting via the Fröhlich Hamiltonian with the bulk LO-phonons of the relevant well material. As such, the fundamental approach followed in this work is to take into account only the generic Q1D aspect of the dynamic behaviour of the electron confined in a free-stand tubular geometry and leave out all the other effects; thus our concern is primarily to give a view of the bulk phonon effects stripped from all other perturbing quantities. Apart from omitting the contributions that may come from all other kinds of phonon mode, we also ignore any screening effects and further complications such as those due to the nonparabolicity corrections to the electron band or the loss of validity of both the effective-mass approximation and the Fröhlich continuum Hamiltonian in thin microstructures. In view of these simplifying assumptions we study the ground state properties (the binding energy, mass and the phonon coupling-induced effective potential) of the Q1D strong-coupling polaron as a function of the coupling strength and the QWW radius.

\section{THEORY}

\subsection{Hamiltonian and wavefunction}

Selecting the phonon quantum $\hbar \omega_{\mathrm{LO}}$ as a unit of energy and $\left(\hbar / 2 m^{*} \omega_{L O}\right)^{1 / 2}$ as a unit of length, the Hamiltonian of a quasi one-dimensional electron immersed in the field of bulk LO-phonons is given by

$$
\begin{aligned}
H= & -\nabla^{2}+\sum_{Q} a_{Q}^{\dagger} a_{Q} \\
& +\sum_{Q} \Gamma_{Q}\left\{a_{Q} \exp (i \boldsymbol{Q} \cdot \boldsymbol{r})+h c\right\},
\end{aligned}
$$

in which $a_{Q}\left(a_{Q}^{\dagger}\right)$ is the phonon annihilation (creation) sperator, and $\overrightarrow{\vec{r}}=(\boldsymbol{Q}, z)$ denotes the electron position in cylindrical coordinates. The interaction amplitude is related to the phonon wavevector $\boldsymbol{Q}=\left(\boldsymbol{q}, q_{z}\right)$ through $\Gamma_{Q}=\sqrt{4 \pi \alpha} / Q$ where $\alpha$ is the coupling constant.

We take the electron trial wavefunction as consisting of two adjustable parameters $\lambda$ and $\mu$ accounting for the anisotropic nature of the confined system

$\Phi_{e}(\varrho, z)=\left(\lambda^{2} / \pi\right)^{1 / 4} \phi(\varrho) \exp \left(-\frac{1}{2} \lambda^{2} z^{2}\right) \mathrm{e}^{i \omega z}$

and

$\phi(\varrho)=n_{\varrho} J_{0}(\kappa \varrho) \exp \left(-\frac{1}{2} \mu^{2} \varrho^{2}\right)$.

In the above, the exponential factor $\mathrm{e}^{i w z}$ (with $\omega$ being a further variational parameter) sets the system in motion, thus enabling one to trace the polaron mass along the wire axis. $J_{0}$ is the zeroth order cylindrical Bessel function of the first kind in which $\kappa=j_{0,1} / R$, where $j_{0,1} \approx 2.4048 \ldots$ is its first zero. The normalisation constant $n_{\varrho}$ is given through $2 \pi \int_{0}^{R} \mathrm{~d} \varrho \varrho \varphi^{2}(\varrho)=1$. With the form of (3) adopted for the lateral part of the electron trial state, the Bessel function takes care of the geometric confinement, and the further confinement induced by phonon coupling is governed by the Gaussian counterpart through parameter $\mu$.

\subsection{Adiabatic formulation}

In the foregoing approximation we assume a highly rapid charge density fluctuation for the electron, to which the lattice responds by acquiring a relaxed static deformation clothing the entire extent of the electron. The adiabatic polaron ground state thus formed can be written in a product ansatz consisting of the electron and lattice parts, i.e.

$\Psi_{g}=\Phi_{e}(\varrho, z) \boldsymbol{U} \mid 0>$

where $\mid 0>$ is the phonon vacuum state, and

$\boldsymbol{U}=\exp \sum_{Q} u_{Q}\left(\Phi_{e}\right)\left[a_{Q}-a_{Q}^{\dagger}\right]$

is the unitary displacement operator changing the reference system of virtual particles by an amount $u_{Q}\left(\Phi_{e}\right)$. It should be noted that simultaneous optimisations with respect to $\Phi_{e}$ and $u_{Q}\left(\Phi_{e}\right)$ correspond to the self-trapping picture of the polaron where the electron distribution and the lattice polarisation influence each other in such a way that a stable relaxed state is eventually attained. Under the canonical transformation $H \rightarrow \boldsymbol{U}^{-1} H \boldsymbol{U}$, equation (1) conforms to

$$
\begin{aligned}
H^{\prime}= & H_{e}+\sum_{Q} u_{Q}^{2}-\sum_{Q} \Gamma_{Q} u_{Q}[\exp (i \boldsymbol{Q} \cdot \boldsymbol{r})+c c] \\
& +\sum_{Q} a_{Q}^{\dagger} a_{Q}+\sum_{Q}\left\{\left[\Gamma_{Q} \exp (i \boldsymbol{Q} \cdot \boldsymbol{r})\right.\right. \\
& \left.\left.-u_{Q}\right] a_{Q}+h c\right\}
\end{aligned}
$$

Since the Hamiltonian is invariant to translations of the electron together with its concomitant lattice distortion, the total momentum along the wire axis

$$
\mathbf{P}_{z}=-i \frac{\partial}{\partial z}+\sum_{Q} q_{z} a_{Q}^{\dagger} a_{Q}
$$

must be conserved. The variation therefore requires an optimisation of the polaron state $\Psi_{g}$, which minimizes $\left\langle\Psi_{g}|H| \Psi_{g}\right\rangle$ subject to the constraint that $\left.<\Psi_{g}\left|P_{z}\right| \Psi_{g}\right\rangle$ is a constant of motion. Thus, minimizing the functional

$$
\begin{aligned}
& \mathrm{F}\left(\lambda, \mu, v_{z} ; \omega, u_{Q}\right) \\
& \equiv<\Phi_{e}\left|\boldsymbol{U}^{-1}\left(H-v_{z} \mathrm{P}_{z}\right) \boldsymbol{U}\right| \Phi_{e}>
\end{aligned}
$$


with respect to $\omega$ and $u_{Q}$ yields

$\omega=\frac{1}{2} v_{z} \quad$ and $\quad u_{Q}\left(\Phi_{e}\right)=\Gamma_{Q} s_{Q} \eta_{Q}$

where

$s_{Q}=<\Phi_{e}|\exp ( \pm i Q \cdot r)| \Phi_{e}>$,

$\eta_{Q}=\left(1-v_{z} q_{z}\right)^{-1}$,

in which the Lagrange multiplier $v_{z}$ is to be identified as the polaron velocity along the wire axis as it turns out (see, e.g. [14]).

In what follows we adopt the case of a stationary polaron, i.e. take $<\Phi_{e}\left|\boldsymbol{U}^{-1} \mathrm{P}_{-} \boldsymbol{U}\right| \Phi_{e}>$ as zero, and thus regard $v_{z}$ as a virtual velocity which we retain in the foregoing steps to keep track of the effective mass of the coupled electron-phonon complex.

In complete form, with the optimal fits for $\omega$ and $u_{Q}$ substituted in equation (8) takes the form

$$
\begin{aligned}
\mathrm{F}\left(\lambda, \mu, v_{z}\right)=\epsilon_{k} & +\sum_{Q} \Gamma_{Q}^{2} s_{Q}^{2}\left(\eta_{Q}^{2}-2 \eta_{Q}\right) \\
& -\frac{1}{4} v_{z}^{2}-\sum_{Q} \Gamma_{Q}^{2} s_{Q}^{2} \eta_{Q}^{2} v_{z} q_{z},
\end{aligned}
$$

where $\epsilon_{k}=<\Phi_{e}\left|-\nabla^{2}\right| \Phi_{e}>$.

In order to trace out the polaron mass from the above equation we have to split the right hand side into its parts consisting of the binding energy of the polaron alone and the additional kinetic contribution, which shows up after having imposed a virtual momentum on the polaron. We are thus tempted to expand the summands in equation (12) in a power series up to order $v_{z}^{2}$. We obtain

$\mathrm{F}\left(\lambda, \mu, v_{z}\right)=E_{g}(\lambda, \mu)-\frac{1}{4} v_{z}^{2} m_{p}$,

where

$E_{g}(\lambda, \mu)=\epsilon_{k}-\sum_{Q} \Gamma_{Q}^{2} s_{Q}^{2}$

refers to the ground state energy and the factor $m_{p}$ multiplying $\frac{1}{4} v_{z}^{2}$ is identified as the polaron mass, given by

$m_{p}=1+4 \sum_{Q} \Gamma_{Q}^{2} s_{Q}^{2} q_{z}^{2}$.

Defining

$$
\begin{aligned}
\sigma_{m m^{\prime}}^{(n)}(x)= & \int_{0}^{j_{0.1}} \mathrm{~d} t t^{n} J_{m}(t) J_{m m^{\prime}}(t) \\
& J_{0}(x t) \exp \left(-\frac{\mu^{2}}{\kappa^{2}} t^{2}\right),
\end{aligned}
$$

we write the following expressions for $\epsilon_{k}$ and $s_{Q}$, which take part in equations (14) and (15)

$\epsilon_{k}=\frac{1}{2} \lambda^{2}+\kappa^{2}+\mu^{2}\left\{2-\frac{2 \sigma_{10}^{(2)}(0)-(\mu / \kappa)^{2} \sigma_{00}^{(3)}(0)}{\sigma_{00}^{(1)}(0)}\right\}$

and

$s_{Q}=r_{q} \exp \left(-\frac{q_{z}^{2}}{4 \lambda^{2}}\right)$,

with

$r_{q}=\frac{\sigma_{00}^{(1)}(q / \kappa)}{\sigma_{00}^{(1)}(0)}$

Projecting out the $Q$-summations: $\sum_{Q} \Gamma_{Q}^{2} s_{Q}^{2} q_{z}^{2 n}$, we further write

$E_{g}(\lambda, \mu)=\epsilon_{k}-\alpha \int_{0}^{\infty} \mathrm{d} q r_{q}^{2} f_{q}$,

and

$m_{p}=1+4 \alpha \int_{0}^{\infty} \mathrm{d} q r_{q}^{2}\left\{\sqrt{\frac{2}{\pi}} \lambda-q f_{q}\right\}$,

where

$f_{q}=\exp \left(\frac{q^{2}}{2 \lambda^{2}}\right) \operatorname{erfc}\left(\frac{q}{\sqrt{2} \lambda}\right)$

with erfe denoting the complementary error function.

\section{RESULTS AND CONCLUSIONS}

In order to obtain the binding energy and effective mass correction of the polaron we numerically minimise equation (20) with respect to the variational parameters $\mu$ and $\lambda$. The parameters thus determined are displayed against the wire radius for a succession of strong $\alpha$ values in Fig. 1(a). It is seen that for large wire radii the curves for $\mu$ and $\lambda$ both have the same asymptotic 3D limit $(\mu=\lambda=\sqrt{2 / 9 \pi} \alpha)$, and as $R$ is made to approach the bulk-polaron size the curves begin to split, depicting the anisotropy due to the confinement imposed by the wire boundary. We note that the place at which the anisotropy starts to show is shifted to smaller $R$ values for stronger phonon coupling, since for large $\alpha$ the starting state of the polaron is already highly localised (as implied by the relatively large values of the parameters $\mu$ and $\lambda$ ) and a smaller-sized polaron feels the effect of the confining boundary only for smaller wire radius.

For a complementary understanding of the variation of the spatial extent of the polaron in the lateral and longitudinal directions, we also provide plots [Fig. 1(b)] of the direct measures of localisation of 
the electron coordinates expressed in terms of the corresponding rms-values given by

$\left.\xi_{\varrho}=\left\{<\Phi_{e}\left|\frac{1}{2} \varrho^{2}\right| \Phi_{e}\right\rangle\right\}^{1 / 2}=\kappa^{-1} \sqrt{\sigma_{00}^{(3)} / \sigma_{00}^{(1)}}$

and

$\xi_{z}=\left\{<\Phi_{e}\left|z^{2}\right| \Phi_{e}>\right\}^{1 / 2}=\left(2 \lambda^{2}\right)^{-1 / 2}$.

It should be noted that the two parameters ( $\alpha$ and $R$ ) characterising the system do not enter the problem in an independent way but together take part in an interrelated manner in the binding, thus inducing an implicit coupling between the transverse and longitudinal coordinates of the electron. Examining the family of curves for $\mu$ and $\lambda$ and for $\xi_{\varrho}$ and $\xi_{z}$ we see that, even though there is no geometric confinement along the wire axis, the axial extent of the polaron shrinks monotonically inward contrary to what one might have expected if the effective electron-LO phonon interaction in the axial direction were insensitive to the variation of $R$.

Going from the bulk case to the quasi-one dimensional limit (Q1D) there comes about a competitive interrelation between whether the charge distribution (and hence the lattice deformation) will condense onto the origin (the polaron centre) or will expand to relax itself in the longitudinal directions along the wire axis.

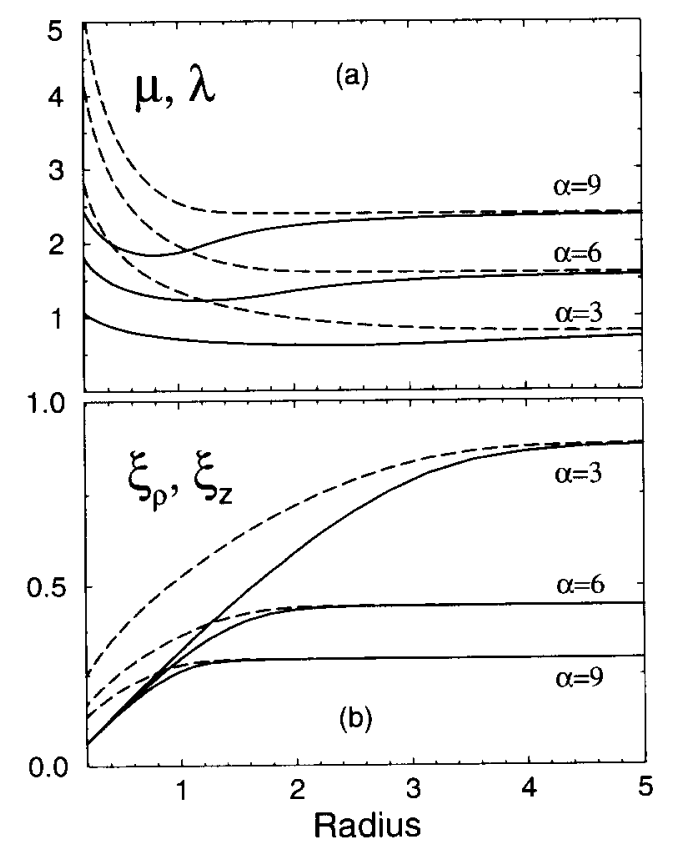

Fig. 1. (a) The variational parameters $\mu$ (solid curve) and $\lambda$ (dashed curve), and (b) the spatial extents $\xi_{o}$ (solid) and $\xi_{-}$(dashed) of the polaron as a function of the wire radius.
Starting from $R \gg 1$ and then restricting the transverse spread of the electron the contribution coming from the tendency of the polaron to expand longitudinally is compensated for by the pseudoenhancement in the effective phonon coupling due to lateral localisation towards the wire axis, thus leading to an overall shrinking spatial extent in the $\pm z$ directions. Meanwhile, with contracting wire size there results an alteration in the lateral structure of the electron wavefunction as depicted by the $\mu$-profile, displaying first a monotonic decrease and then an increase, implying that the radial part, $\phi(\varrho)$, of the electron wavefunction conforms to a form structured more by its Bessel-function counterpart, $J_{0}(\kappa \varrho)$, rather than a narrow Gaussian, $\exp \left(-\mu^{2} \varrho^{2} / 2\right)$, decaying far before the boundary is reached (cf., Fig. 2). This can alternatively be recognised from the fact that, regardless of $\alpha$, the curves for $\xi_{\varrho}$ [Fig. 1(b)] all tend to the same asymptote, meaning that at small wire radii the lateral extent of the polaron is governed mainly by the geometric confinement rather than phonon coupling-induced localisation. A complementary feature is that when $R$ is far below unity both $\mu$ and $\lambda$ display rather rapidly growing profiles compatible with a considerably pronounced effective phonon coupling and a highly localised characterisation of the polaron in all directions.

For completeness, we also present a pictorial view

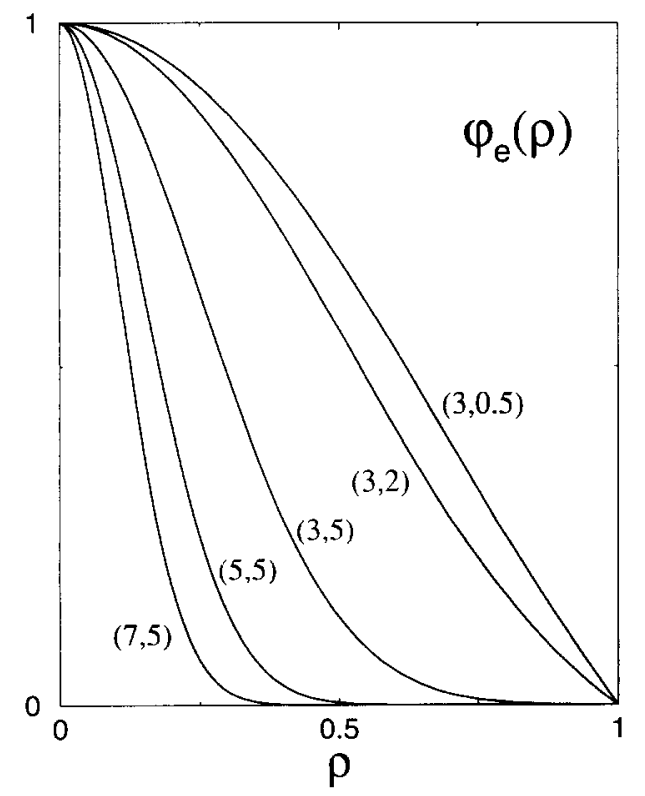

Fig. 2. $\varphi(\varrho)$ versus $\varrho$ for various pairs $(\alpha, R)$ of $\alpha$ $(=3,5,7)$ and $R(=0.5,2,5)$. In the plots the peak value of $\phi$ is normalised to unity, and $\varrho$ is expressed in units of $R$. 


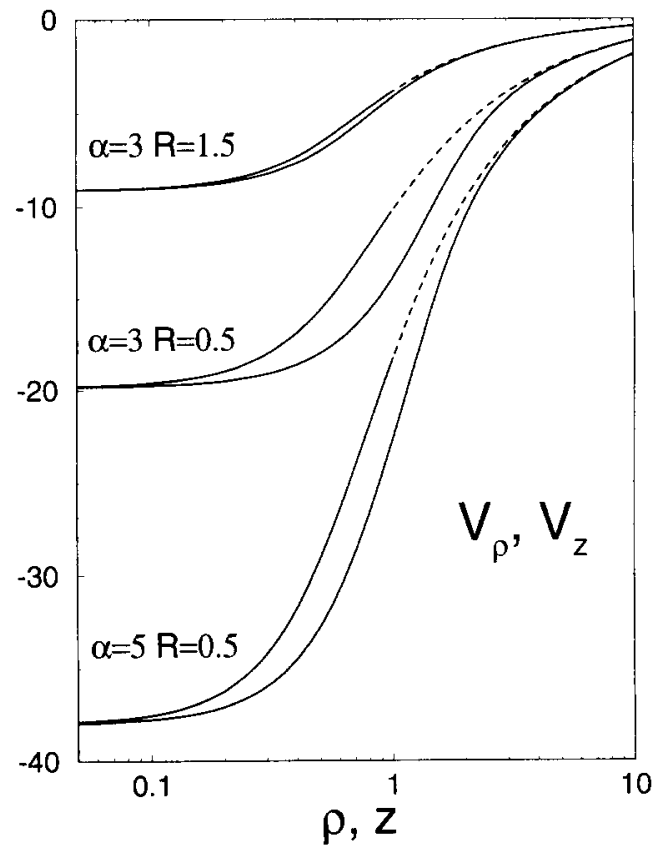

Fig. 3. The phonon coupling-induced potential well profiles in the radial and longitudinal directions represented, respectively, by the upper and lower curves. The potentials $V_{o}$ and $V_{z}$ are given in arbitrary units on a linear scale, whereas $\varrho$ and $z$ are expressed in terms of $R$.

of the phonon-coupling-induced potential well profiles

$V(\varrho, z)=\frac{1}{e} \sum_{Q} \Gamma_{Q}<-0\left|\mathbf{U}^{-1}\left(\mathrm{e}^{i \boldsymbol{Q} \cdot r} a_{Q}+h c\right) \mathbf{U}\right| 0>$,

along the radial and transverse directions (cf. Appendix). In Fig. 3 we plot $V_{\varrho} \equiv V(\varrho, z=0)$ and $V=\equiv V(\varrho=0, z)$ for different permutations of $\alpha$ $(=3,5)$ and $R(0.5,1.5)$. It is readily seen that the interaction potential gets deeper for strong $\alpha$ and/or narrow $R$ where the two parameters together play a combined role in favour of a more effective coupling of the electron to the phonon field. The spatial anisotropy mentioned in the preceding paragraph is also portrayed in the set of curves for the potential profiles in that $V_{z}$ lies deviated below $V_{\varrho}$, the digression being most significant for small $R$ values and at sites more on the boundary side rather than the axial region $(\varrho / R, z \mid R \leq 0.1)$ where $V_{\varrho}$ and $V_{z}$ join and form spherically symmetric (isotropic) equipotentials.

A more brief content of the arguments given above is provided in Fig. 4 where we plot the binding energy, $\epsilon_{p}=\left(j_{0,1} / R\right)^{2}-E_{g}$ (relative to the subband), and the polaronic contribution to the band mass, $\Delta m_{p}=m_{p}-1$, against the wire radius for a set of

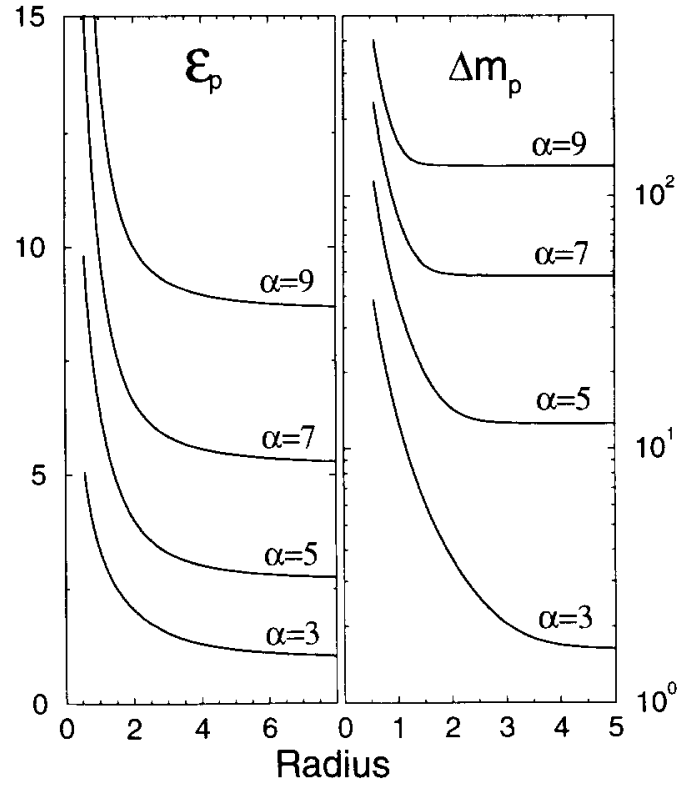

Fig. 4. $\epsilon_{p}$ and $\Delta m_{p}$ as functions of $R$.

distinctive $\alpha$-values. We once again note the same qualitative behaviour where the growth rates of $\epsilon_{p}$ and $m_{p}$ are somewhat moderate for large $R$, but however on the opposite extreme where $R$ is tuned to smaller values both $\epsilon_{p}$ and $m_{p}$ are observed to increase with very pronounced slopes the growth rates, which are significantly greater for stronger $\alpha$.

The adiabatic theory employed in this work gives $\epsilon_{p}^{(3 D)}=\alpha^{2} / 3 \pi$ in the bulk case and $\epsilon_{p}^{(2 D)}=(\pi / 8) \alpha^{2}$ for a strictly two-dimensional polaron [15]. The general trend that the polaron quantities are inherently pronounced in low dimensional systems is also reflected in our present studies. For a wire with $\alpha=3$ and $R=1$, for instance, we obtain $\epsilon_{p}=3.377$. For thinner wires the binding gets naturally deeper since the electronic wavefunction becomes even more localised in all directions perpendicular to the wire axis. We obtain $\epsilon_{p}=5.485$ when $R=0.5$ and $\epsilon_{p}=9.920$ when $R=0.2$. A comparison of these values with the corresponding three- $\left(\epsilon_{p}^{(3 D)}=0.955\right)$ and two$\left(\epsilon_{p}^{(2 D)}=3.534\right)$ dimensional values reveals that the polaron binding energy is much greater when the effective dimensionality is reduced from three to one than when reduced from three to two. The same is true for the polaron mass where $\Delta m_{p}$ in quantum wires of small dimensions are much larger than those in comparable two-dimensional wells.

\section{REFERENCES}

1. H. Sakaki, J. Appl. Phys. 19, L735 (1980).

2. P.M. Petroff, A.C. Gossard, R.A. Logan \& W.W. Wiegmann, Appl. Phys. Lett. 41, 635 (1982). 
3. T.J. Thornton, M. Pepper, H. Ahmed, D. Andrews \& G.J. Davies, Phys. Rev. lett. 56, 1198 (1986).

4. H. Temkin, G.J. Dolan, M.B. Parish \& S.N.G. Chu, Appl. Phys. Lett. 40, 413 (1987).

5. R.C. Tonucci, B.L. Justus, A.J. Campillo \& C.E. Ford, Science 258, 783 (1992).

6. J.P. Leburton, J. Appl. Phys. 56, 2850 (1984).

7. M.H. Degani \& O. Hipólito, Solid State Commun. 65, 1185 (1988).

8. N.C. Constantinou \& B.K. Ridley, J. Phys. Condens. Matter 1, 2283 (1989).

9. M.H. Degani \& G.A. Farias, Phys. Rev. B42, 11950 (1990).

10. T. Yildirim \& A. Erçelebi, J. Phys. Condens. Matter 3, 1271 (1991).

11. T. Yildirim \& A. Erçelebi, J. Phys. Condens. Matter 3, 4357 (1991).

12. H.-Y. Zhou \& S.-W. Gu, Solid State Commun. 91, 725 (1994).

13. B. Tanatar, J. Phy's. Condens. Matter 5, 2203 (1993).

14. G.D. Whitfield, R. Parker \& M. Rona, Phys. Rev. B13, 2132 (1976).

15. S. Das Sarma \& B.A. Mason, Ann. Phys., NY 163, 78 (1985).

\section{APPENDIX}

Using equation (6) with $u_{Q}=\Gamma_{Q} s_{Q}$, equation (25) conforms to

$V(\varrho, z)=-\frac{1}{e} \sum_{Q} \Gamma_{Q}^{2} s_{Q}\left(\mathrm{e}^{i Q \cdot r}+c c\right)$,

in which $s_{Q}$ is given by equation (18). Setting $z=0$ and $\varrho=0$, respectively, for the potential profiles along the radial $(\varrho)$ - and longitudinal ( $z$ )-directions and projecting out the wavevector sum we obtain

$V_{\varrho}=-\frac{2}{e} \alpha \int_{0}^{\infty} \mathrm{d} q r_{q} \mathrm{e}^{t^{2}} \operatorname{erfc}(t) J_{0}(\varrho q)$,

$V_{z}=-\frac{1}{e} \alpha \int_{0}^{\infty} \mathrm{d} q r_{q} \mathrm{e}^{t^{2}}\left[h_{q}(z)+h_{q}(-z)\right]$,

where

$t=\frac{q}{2 \lambda} \quad$ and $\quad h_{q}(z)=\mathrm{e}^{-q} \operatorname{erfc}(t+\lambda z)$. 